\title{
Nephrology and Coronavirus Disease 2019
}

\author{
Joyita Bharati Raja Ramachandran ${ }^{1}$ \\ ${ }^{1}$ Department of Nephrology, Post Graduate Institute of Medical \\ Education and Research, Chandigarh, India
}

\begin{abstract}
Address for correspondence Raja Ramachandran, DM, Department of Nephrology, Post Graduate Institute of Medical Education and Research, Chandigarh 160012, India (e-mail: drraja_1980@yahoo.co.in).
\end{abstract}

Ann Natl Acad Med Sci (India):2020;3:166-170

\begin{abstract}
Keywords

- COVID-19

- end-stage renal disease

- acute kidney injury

- continuous renal replacement therapy

Novel coronavirus disease 2019 (COVID-19) has emerged as a threat to the human population worldwide. Old age and presence of comorbidities are risk factors for severe complications of the disease, as many of these patients are not able to mount effective or have an aberrant antiviral response. Patients with chronic kidney disease are at high risk of COVID-19 and its complications, especially those with end-stage renal disease (ESRD). Patients have repeated exposure to crowding in dialysis units, and face the uncertainties of health care system sustainability during periods of increasing demand and scarce supply. Acute kidney injury (AKI) is a notable complication in patients with COVID-19 with an incidence of up to $25 \%$ in specific populations. Patients with AKI have a higher mortality rate. The etiology and pathogenesis of AKI in COVID-19 are multifactorial. Direct viral invasion and cytopathic effects on renal tubular cells and indirect factors like hypovolemia, rhabdomyolysis, renal vein congestion, and hypercoagulable state play a role in causing AKI in these patients. Renal replacement therapy in the form of continuous renal replacement therapy is most widely used worldwide mostly due to hemodynamic instability of critically sick patients with COVID-19. Anticoagulation therapy is critical as most COVID-19 patients have increased clotting tendency and extracorporeal circuit thrombosis is common. ESRD patients in China had mild-to-moderate symptoms commonly. However, the data from Spain and Italy report a higher mortality rate in ESRD patients than the general population. Strict screening and uniform adherence to infection control practices have led to a decreased risk of cross-infection among other patients and dialysis personnel in dialysis units. The association between nephrology and COVID-19 is unique and focus on further research on kidney pathology along with the strengthening of existing dialysis facilities are needed.
\end{abstract}

\section{Introduction}

The infection caused by severe acute respiratory distress syndrome coronavirus 2 (SARS- CoV-2), also called the coronavirus disease 2019 (COVID-19), is closely linked to the kidneys, both in terms of commonly affecting the kidneys and having an increased prevalence and severity in patients with chronic kidney diseases (CKDs)., ${ }^{1,2}$ Unlike the previous pandemics caused by respiratory viruses, the SARS-CoV-2 virus affects multiple organ systems apart from severe respiratory involvement and spreads quickly among healthy asymptomatic individuals. The presence of comorbidities namely hypertension, diabetes mellitus, CKD, and immunosuppressed state namely cancer and organ transplant recipients have clearly emerged to be the risk factors for life-threatening COVID-19.,4

\section{Pathogenesis of Coronavirus Disease 2019 Related Kidney Disease}

SARS-CoV-2 can affect the kidneys both directly and indirectly. Kidney involvement can be in the form of acute 
kidney injury (AKI), proteinuria, and hematuria. ${ }^{2}$ AKI is typically seen in severe COVID-19 disease owing to multiple mechanisms. ${ }^{5}$ Direct viral (SARS-CoV-2) invasion in kidneys, specifically the renal tubular epithelium and podocytes, was demonstrated in a postmortem study in Chinese patients with severe COVID-19 pneumonia. ${ }^{6}$ Virus-like particles were shown ultrastructurally and SARS-CoV-2 nucleoprotein by indirect immunofluorescence using an antibody. ${ }^{6}$ Direct viral infection could be associated with AKI and proteinuria in these patients as it correlates with histopathological findings of tubular injury in the form of loss of brush border and vacuolation in the proximal tubular epithelial cells along with podocyte vacuolation with occasional detachment. In another preprint study in medRxiv, ${ }^{7}$ virus-like particles in the tubular epithelium-in addition to a direct cytopathic effect-were shown to initiate macrophage and complement deposition which cause tubular injury. However, lack of demonstration of SARS-CoV-2 RNA and lack of inclusion of patients with AKI who had less severe symptoms of COVID-19, make a case for further indagating to understand kidney injury in COVID-19. Indirect causes of AKI due to hypoxic or toxin mediated acute tubular injury are often common in patients with severe COVID-19. ${ }^{5}$ Cytokine release syndrome (cytokine storm), driven predominantly by interleukin (IL)-6, is one among the various culprits. ${ }^{5}$ Systemic endothelial injury, increased vascular permeability, intravascular hypovolemia, and therefore renal tubular injury are the harbinger to the cytokine storm. Cytokine release syndrome is also frequently associated with cardiomyopathy which results in renal tubular injury by similar mechanisms as cardiorenal syndrome type $1 .{ }^{5}$ Occurrence of rhabdomyolysis in critically sick patients on mechanical ventilation is also attributed to cause AKI in COVID-19 disease. Histopathological findings of hemosiderin granules in tubular epithelium and occasional pigmented casts in tubular lumen of few patients with elevated creatine phosphokinase indicate rhabdomyolysis as a contributing factor for AKI. ${ }^{6}$ Renal compartment syndrome-secondary to ventilatory abnormalities or fluid overload from aggressive fluid resuscitation in patients with severe COVID-19-could lead to increased renal vein congestion and impaired venous drainage and therefore AKI. ${ }^{5,8,9}$ It is also possible that renal infarction due to hypercoagulable state seen in COVID-1910 could also result in acute tubular or cortical necrosis. ${ }^{11}$ The various possible pathogenetic mechanisms of AKI in patients with COVID-19 are shown in - Table $\mathbf{1}$.

The histopathological findings of the kidneys include acute tubular injury (in various forms) and occasional podocytopathy. ${ }^{6}$ The vascular (peritubular capillaries and glomerular capillary loops) involvement in the form of aggregation of red blood cells which are devoid of fibrin or platelet and are associated with surrounding secondary endothelial injury ${ }^{6}$ The reason for this finding is unclear, and the absence of a typical thrombus (as seen in thrombotic microangiopathy) and interstitial hemorrhage (as seen in Hantavirus infection) suggest need for future deliberation on COVID-19 related kidney pathology. Only 3 out of 26 patients had evidence of few segmental fibrin thrombi in the glomerular capillary loops along with endothelial injury. ${ }^{6}$ Disseminated
Table 1 Direct and indirect causes of acute kidney injury in coronavirus disease 2019

\begin{tabular}{|l|}
\hline Direct cause \\
\hline $\begin{array}{l}\text { Direct viral invasion (likely) causing cytopathic effects on } \\
\text { renal tubular cells and podocytes }\end{array}$ \\
\hline Indirect causes \\
\hline Hypoxic acute tubular injury \\
i. Secondary to hypovolemia and decreased perfusion \\
ii. $\quad$ Cytokine release syndrome \\
iii. $\quad$ Cardiorenal syndrome type 1 \\
\hline Rhabdomyolysis-mediated acute tubular injury \\
i. Secondary to hyperventilation related muscle injury \\
\hline Renal compartment syndrome \\
i. Secondary to renal vein congestion \\
ii. $\quad$ Ventilation associated increased abdominal pressure \\
iii. $\quad$ Aggressive fluid resuscitation \\
\hline $\begin{array}{l}\text { Renal infarction } \\
\text { i. } \quad \text { Secondary to hypercoagulable state }\end{array}$
\end{tabular}

intravascular coagulation as manifested by elevated D-dimer and/or low platelet counts) was seen in a majority, whereas significant clinical renal dysfunction (elevated serum creatinine $>1.2 \mathrm{mg} / \mathrm{dL}$ ) was not universally present in these patients despite having pathological evidence of acute tubular injury. ${ }^{6}$ The high frequency of expression of angiotensin converting enzyme 2 (ACE2), required for SARS-CoV-2 to enter host cells, on tubular epithelial cells and podocytes suggest that viral entry in the kidneys is facilitated. ${ }^{6}$ Although glomerular abnormalities are not described commonly in the limited available literature on kidney pathology in COVID-19, collapsing glomerulopathy was reported recently in an African-American woman with high-risk APOL1 allele who developed rapid decline in kidney function in the presence of COVID-19. ${ }^{12}$ While direct infection of kidneys was not found, presence of COVID-19 was hypothesized to be a second hit for the development of collapsing glomerulopathy in a predisposed individual. ${ }^{12}$

\section{Acute Kidney Injury in Coronavirus Disease 2019}

The incidence of AKI among all patients with proven COVID-19 varied from $0.5^{3}$ to $11 \%^{2,13-15}$ in China and 19.1 to $22 \%$ in the United States, respectively. ${ }^{16,17}$ Further, when only critically sick patients with COVID-19 pneumonia were studied, AKI was documented in $29 \%$ in China. ${ }^{18}$ Notably, AKI is prevalent in intensive care units (ICUs) caring for critically sick patients with COVID-19 and is associated with poor patient survival. ${ }^{2,18}$ Several investigational therapies are ongoing clinical trials and hopefully will be able to be a potential cure for patients affected by COVID-19 and its complications such as AKI. The requirement of renal replacement therapy (RRT) was reported in $17 \%$ of all critically sick patients with COVID-19 in China. ${ }^{18}$ Further, based on current evidence, the timing and modality of RRT in these patients is no different from any 
other similar patient in the ICU. However, experts suggest the use of convection-based dialysis modalities in such patients for taking care of the "cytokine storm." Hemoperfusion and continuous renal replacement therapy (CRRT) using adsorptive filters are proposed to be beneficial for the removal of large cytokines such as interleukin- $6 .^{5}$ There is no conclusive clinical evidence for choosing one modality such as CRRT over slow low efficiency dialysis in COVID-19 patients. Hemodynamic tolerability, resource availability, and local expertise would guide therapy in most situations outside of clinical trials. Extracorporeal blood circuit clotting is another common finding in critically sick COVID-19 patients undergoing RRT due to the hypercoagulable state. Appropriate and early anticoagulation along with dynamic monitoring are crucial. Peritoneal dialysis (PD) is beneficial in this regard specifically. In the absence of hypercatabolic state and difficult ventilation in a patient with COVID-19 requiring RRT, acute PD has advantages in the form of no requirement of vascular access and extracorporeal circuit, cardiovascular stability, minimal requirement for infrastructure and trained dialysis personnel and to an extent, cost-effectiveness. While delivering the best therapy is an important concern, being able to deliver any therapy to all patients is a parallel concern in the current stage of this pandemic. In this regard, the American Society of Nephrology has recommended shortening of sessions of CRRT to 10 hours by augmenting flow rates $(40-50 \mathrm{~mL} / \mathrm{kg} /$ hour $)$, if need be, so as to provide CRRT to another patient. ${ }^{19}$ The summary of AKI in various clinical reports is shown in $\boldsymbol{- T a b l e} \mathbf{2}$.

\section{Chronic Kidney Disease and Coronavirus Disease 2019}

Elderly age and presence of chronic comorbidities have been shown to be associated with increased risk of severe COVID-19 infection., ${ }^{3,4}$ Among all chronic comorbidities, diabetes, and cardiovascular diseases are common and CKD is described in approximately 1 to $4.7 \%$ of patients.,4,16,18 In a prospective study in admitted Chinese patients with COVID-19, baseline kidney abnormalities at admissionin the form of proteinuria or hematuria or elevated serum creatinine-were associated with increased risk of in-hospital death. ${ }^{2}$ Further, elevated baseline creatinine was associated with increased risk of AKI and increased likelihood of admission to ICU. ${ }^{2}$ The authors concluded that patients with kidney abnormalities (in any form) should be aggressively evaluated further for progressive disease. Patients with CKD are immunosuppressed and hence at an increased risk of upper respiratory tract infections. Moreover, CKD patients often have other comorbidities such as diabetes, hypertension, and cardiovascular diseases which are added risk factors for COVID-19. Patients with diabetic kidney disease have been shown to have altered expression of ACE2 and ACE messenger ribonucleic acid as compared with healthy controls. ${ }^{20}$ This could predispose such patients to higher risk of AKI. Despite the link between ACE2 and SARS-CoV-2 infection, there is no evidence to prove that continuing the use of ACE inhibitor or angiotensin receptor blocker receptor (ARB) in patients with COVID-19 is associated with risk of acquiring or having severe COVID-19 infection or death. ${ }^{21}$ The American college of cardiology ${ }^{22}$ and the European society of cardiology ${ }^{23}$ have given similar statements in this regard advising patients to continue the use of ACE inhibitor or ARB during this pandemic.

\section{End-Stage Renal Disease and Coronavirus Disease 2019}

Patients on chronic hemodialysis are at higher risk of both acquiring and having severe COVID-19 as they are often oldaged, have concomitant comorbidities, and need to gather in crowded dialysis facilities frequently. Hemodialysis patients are more prone to bear the brunt of this pandemic as dialysis units, the life-line for majority of ESRD, are facing an unique challenge among all other health care systems. ${ }^{24} \mathrm{Hemodialysis}$ patients in China were shown to have lesser peripheral blood inflammatory cytokines and milder symptoms of COVID-19 than other COVID-19 patients in a preprint form in medRxiv. ${ }^{25}$ Further, in another study from China, the incidence of COVID-19 among all patients on maintenance hemodialysis was 2.15 and $77 \%$ of patients had mild-to-moderate symptoms, with fever seen in $51 \%{ }^{26}$ Universal screening

Table 2 Incidence of acute kidney injury and use of renal replacement therapy in coronavirus disease 2019 patients in reported studies

\begin{tabular}{|c|c|c|c|}
\hline Author (year) & Incidence of AKI & Use of RRT & Risk factors for AKI \\
\hline Huang et al ${ }^{15}(2020)$ & $7 \%$ & $7 \%$ & NA \\
\hline Chen et al ${ }^{14}(2020)$ & $11 \%$ & $1 \%$ & NA \\
\hline Cheng et $\mathrm{al}^{2}(2020)$ & $5.1 \%$ & NA (AKI stage 3 in $2 \%$ ) & Elevated baseline serum creatinine \\
\hline Guan et $\mathrm{al}^{3}$ (2020) & $0.5 \%$ & $0.8 \%$ & NA \\
\hline Wu et $\mathrm{al}^{13}(2020)$ & $4.5 \%$ & NA & NA \\
\hline Yang et al ${ }^{18}(2020)$ & $29 \%$ & $17 \%$ & NA \\
\hline Richardson et al ${ }^{16}(2020)$ & $22 \%$ & $3.2 \%$ & Diabetes mellitus \\
\hline Arentz et al, ${ }^{17}$ (2020) & $19.1 \%$ & NA & NA \\
\hline
\end{tabular}

Abbreviations: AKI, acute kidney injury; NA, not available; RRT, renal replacement therapy. 
with chest computed tomography (CT) showed lung finding even in asymptomatic patients which constituted $21.4 \%$. This amplifies the possibility of rapid spread of COVID-19 in dialysis centers as majority of patients can be asymptomatic or mildly symptomatic and missed on routine screening. In such a situation, transmission to health care workers in dialysis units is another challenge as the working of the unit depends on their skilled expertise. Reports from Spain and Italy found high mortality rates (30.5 and 25\%, respectively) in ESRD patients with COVID-19.27,28 Dialysis vintage, lymphopenia, and increased lactate dehydrogenase at admission were predictors of mortality. ${ }^{27}$ Of note, three (8.3\%) patients had documented nosocomial transmission. ${ }^{27}$ While CT-based screening of all ESRD patients was found to detect asymptomatic ESRD patients, nonspecificity of CT findings in ESRD patients, nonavailability of CT scan facilities in many centers, and concerns over wider exposure of a potential patient to hospital staff while taking up to imaging room are some of its limitations. Treatment of most of the ESRD patients with COVID-19 included antivirals (lopinavir/Ritonavir) in China and antivirals with hydroxychloroquine in Spain and Italy. ${ }^{26-28}$ Recommendations by professional societies internationally (America ${ }^{29}$ and Europe ${ }^{30}$ ) and nationally ${ }^{31}$ have focused on temperature and symptom screening, and not radiology based screening, at the entrance of hemodialysis units. Health care facilities should implement changes to the functioning of the existing dialysis unit including universal use of medical grade masks by patients, provision of adequate personal protective equipment to dialysis staff and educating them regularly, apart from strict screening, so as to take care of potential asymptomatic ESRD patients. Moreover, separate area for COVID-19 suspected patients should be made to quickly isolate such patients. Recommendations on strategies to contain COVID-19 among dialysis centers are available from professional societies from America, ${ }^{29}$ Europe, ${ }^{30}$ China, ${ }^{32}$ and India. ${ }^{31}$

\section{Kidney Transplant Recipients and Coronavirus Disease 2019}

Kidney transplant recipients are at heightened risk for severe COVID-19 due to their immunosuppressed state. Case reports and series describe severe COVID-1933 and atypical manifestations in transplant recipients. ${ }^{34}$ In a cohort of 15 transplant patients hospitalized with COVID-19 in New York, ${ }^{35}$ clinical presentation was akin to the general population, and 53\% had a successful recovery. The incidence of AKI was higher (40\%) than the general population (up to 25\%). However, another study comprising of 20 patients from Italy reported a rapid progression of pneumonia and a mortality rate of $25 \%{ }^{36}$ The optimal management of immunosuppression is not known. However, a reduction in the dose or discontinuation of antimetabolites and sometimes, calcineurin inhibitors are the current consensus. ${ }^{35,36}$ Various therapies, namely antivirals, hydroxychloroquine, interferon- $\alpha$, and tocilizumab remain nugatory. ${ }^{35,36}$ An attempt to reintroduce immunosuppressive agents at 2 weeks postdischarge is currently practiced in New York. ${ }^{35}$

To conclude, the association between COVID-19 and nephrology is unique. Future research needs to deliberate on the clinical characteristics and pathological findings of COVID-19 related kidney injury for understanding the implications of evaluation for kidney involvement in COVID-19. The current pandemic also exposes the fault lines in the health care delivery worldwide and holler for the longterm strengthening of the existing dialysis facilities as a priority among health care imperatives.

\section{Conflict of Interest}

None declared.

\section{References}

1 Su H, Yang M, Wan C, et al. Renal histopathological analysis of 26 postmortem findings of patients with COVID-19 in China. Kidney Int 2020. Doi: 10.1016/j.kint.2020.04.003

2 Cheng Y, Luo R, Wang K, et al. Kidney disease is associated with in-hospital death of patients with COVID-19. Kidney Int 2020;97(5):829-838

3 Guan WJ, Ni ZY, Hu Y, et al; China Medical Treatment Expert Group for Covid-19. Clinical Characteristics of Coronavirus Disease 2019 in China. N Engl J Med 2020;382(18):1708-1720

4 Grasselli G, Zangrillo A, Zanella A, et al; COVID-19 Lombardy ICU Network. Baseline characteristics and outcomes of 1591 patients infected with SARS-CoV-2 admitted to ICUs of the Lombardy region, Italy. JAMA 2020;323(16):1574-1581

5 Ronco C, Reis T. Kidney involvement in COVID-19 and rationale for extracorporeal therapies. Nat Rev Nephrol 2020;16(6):308-310

6 Su H, Yang M, Wan C, et al. Renal histopathological analysis of 26 postmortem findings of patients with COVID-19 in China. Kidney Int 2020;98(1):219-227

7 Diao B, Wang $\mathrm{CH}$, Wang RS, et al. Human kidney is a target for novel severe acute respiratory syndrome coronavirus 2. Available at: https://www.medrxiv.org/content/10.1101/20 20.03.04.20031120v4. Accessed April 10, 2020

8 Bailey J, Shapiro MJ. Abdominal compartment syndrome. Crit Care 2000;4(1):23-29

9 Claure-Del Granado R, Mehta RL. Fluid overload in the ICU: evaluation and management. BMC Nephrol 2016;17(1):109

10 Tang N, Li D, Wang X, Sun Z. Abnormal coagulation parameters are associated with poor prognosis in patients with novel coronavirus pneumonia. J Thromb Haemost 2020;18(4):844-847

11 Batlle D, Soler MJ, Sparks MA, et al; COVID-19 and ACE2 in Cardiovascular, Lung, and Kidney Working Group. Acute kidney injury in COVID-19: emerging evidence of a distinct pathophysiology. J Am Soc Nephrol 2020. Doi: 10.1681/ ASN.2020040419

12 Larsen CP, Bourne TD, Wilson JD, Saqqa O, Sharshir MA. Collapsing glomerulopathy in a patient with coronavirus disease 2019 (COVID-19) Kidney Int Rep 2020. Doi: 10.1016/j. ekir.2020.04.002

13 Wu C, Chen X, Cai Y, et al. Risk factors associated with acute respiratory distress syndrome and death in patients with coronavirus disease 2019 pneumonia in Wuhan, China. JAMA Intern Med 2020. Doi: 10.1001/jamainternmed.2020.0994

14 Chen $\mathrm{T}$, Wu D, Chen $\mathrm{H}$, et al. Clinical characteristics of 113 deceased patients with coronavirus disease 2019: retrospective study. BMJ 2020;368:m1091 
15 Huang C, Wang Y, Li X, et al. Clinical features of patients infected with 2019 novel coronavirus in Wuhan, China. Lancet 2020;395(10223):497-506

16 Richardson S, Hirsch JS, Narasimhan M, et al; and the Northwell COVID-19 Research Consortium. Presenting characteristics, comorbidities, and outcomes among 5700 patients hospitalized with COVID-19 in the New York City Area. JAMA 2020. Doi: $10.1001 /$ jama.2020.6775

17 Arentz M, Yim E, Klaff L, et al. Characteristics and outcomes of 21 critically ill patients with covid-19 in Washington state. JAMA 2020;323(16):1612-1614

18 Yang X, Yu Y, Xu J, et al. Clinical course and outcomes of critically ill patients with SARS-CoV-2 pneumonia in Wuhan, China: a single-centered, retrospective, observational study. Lancet Respir Med 2020;8(5):475-481

19 American Society of Nephrology. Recommendations On The Care Of Hospitalized Patients With Covid-19 And Kidney Failure Requiring Renal Replacement Therapy. Available at: https://www. asn- online.org/g/blast/files/AKI_COVID19_Recommendations_ Document_03.21.2020.pdf. Accessed March 21, 2020

20 Mizuiri S, Hemmi H, Arita M, et al. Expression of ACE and ACE2 in individuals with diabetic kidney disease and healthy controls. Am J Kidney Dis 2008;51(4):613-623

21 JarchoJA, Ingelfinger JR, Hamel MB, D'Agostino RBSr, Harrington DP. Inhibitors of the Renin-Angiotensin-Aldosterone System and Covid-19. N Engl J Med 2020;382(25):2462-2464

22 American College of Cardiology. HFSA/ACC/AHA statement addresses concerns re: using RAAS antagonists in COVID-19. Available at: https://www.acc.org/latest-in-cardiology/articles/2020/03/17/08/59/hfsa-acc-aha-statement-addressesconcerns- re-using-raas-antagonists-in-covid-19. Accessed March 17, 2020

23 European Society of Cardiology. Position statement of the ESC Council on Hypertension on ACE-inhibitors and angiotensin receptor blockers. Available at: https://www.escardio.org/Councils/ Council-on-Hypertension- (CHT)/News/position-statement-ofthe-esc-council-on-hypertension-on-ace- inhibitors-and-ang. opens in new tab. Accessed March 13, 2020

24 Ramachandran $\mathrm{R}$, Jha V. Adding insult to injury: kidney replacement therapy during COVID-19 in India. Kidney Int 2020. Doi: 10.1016/j.kint.2020.04.019

25 Ma Y, Diao B, Lv X, et al. 2019 Novel coronavirus disease in hemodialysis (HD) patients: Report from one HD center in Wuhan, China. Available at: https://www.medrxiv.org/conten t/10.1101/2020.02.24.20027201v3. Accessed June 17, 2020
26 Xiong F, Tang H, Liu L, et al. Clinical characteristics of and medical interventions for COVID-19 in hemodialysis patients in Wuhan, China. J Am Soc Nephrol 2020. Doi: 10.1681/ ASN.2020030354

27 Goicoechea M, Camara LAS, Macias N, et al. COVID-19: clinical course and outcomes of 36 hemodialysis patients in Spain. Kidney Int 2020. Doi: 10.1016/j.kint.2020.04.031

28 Alberici F, Delbarba E, Manenti C, et al; Brescia Renal COVID Task Force. Management of patients on dialysis and with kidney transplantation during the SARS-CoV-2 (COVID-19) pandemic in Brescia, Italy. Kidney Int Rep 2020;5:580-585

29 American Society of Nephrology. Nephrologists transforming dialysis safety, coronavirus disease 2019. Available at: https:// www.asn-online.org/ntds/. Accessed June 26, 2020

30 Basile C, Combe Christian, Pizzarelli F, et al. on behalf of the EUDIAL Working Group of ERA-EDTA, Recommendations for the prevention, mitigation and containment of the emerging SARSCoV-2 (COVID-19) pandemic in haemodialysis centres. Available at: https://doi.org/10.1093/ndt/gfaa069. Accessed March 20, 2020

31 MOHFW guidelines for dialysis of COVID-19 patients. Available at: https://www.mohfw.gov.in/pdf/Guidelinesfor DialysisofCovid19Patients.pdf. Accessed June 26, 2020

32 Expert Team of Chinese Medical Association Nephrology Branch. Recommendations for prevention and control of novel coronavirus infection in blood purification center (room) from Chinese Medical Association Nephrology Branch. Chin J Nephrol. 2020;36:82-84

33 Banerjee D, Popoola J, Shah S, Ster IC, Quan V, Phanish M. COVID-19 infection in kidney transplant recipients. Kidney Int 2020;97(6):1076-1082

34 Guillen E, Pineiro GJ, Revuelta I, et al. Case report of COVID-19 in a kidney transplant recipient: Does immunosuppression alter the clinical presentation? Am J Transplant 2020. Doi: $10.1111 /$ ajt.15874

35 Columbia University Kidney Transplant Program. Early description of coronavirus 2019 disease in kidney transplant recipients in New York. J Am Soc Nephrol 2020;31(6):1150-1156

36 Alberici F, Delbarba E, Manenti C, et al. A single center observational study of the clinical characteristics and short-term outcome of 20 kidney transplant patients admitted for SARS-CoV2 pneumonia. Kidney Int 2020;97(6):1083-1088 